\title{
GMR
}

\section{Comparative analysis of phylogenetic relationships, morphologies, and pathogenicities among Curvularia lunata isolates from maize in China}

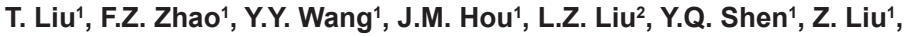 \\ H.T. Zhang ${ }^{1}$ and Y.H. Zuo ${ }^{1}$ \\ ${ }^{1}$ Institute of Plant Pathology and Applied Microbiology, Agricultural School, \\ Heilongjiang Bayi Agricultural University, Daqing, Heilongjiang, China \\ ${ }^{2}$ Shanghai Academy of Agricultural Sciences, Shanghai, China \\ Corresponding author: Y.H. Zuo \\ E-mail: liutongamy@sina.com
}

Genet. Mol. Res. 14 (4): 12537-12546 (2015)

Received May 13, 2015

Accepted July 31, 2015

Published October 16, 2015

DOI http://dx.doi.org/10.4238/2015.October.16.21

ABSTRACT. To understand the effects of disease-resistant maize varieties and new cropping systems on the population of Curvularia lunata, 52 isolates of $C$. lunata were collected in China from 2011 to 2013. The isolates were analyzed in terms of phylogenetic relationships, morphology, and pathogenicity. Phylogenetic analysis showed that the 52 isolates clustered into 2 distinct clusters with further subdivisions, suggesting the emergence of new genetic divergence within C. lunata. Results of morphology and pathogenicity analyses demonstrated that there were significant differences among these isolates: 27 isolates were classified as fast growing, 5 as slow growing, and 20 as moderate growing. Three isolates had white-colored colonies, 13 had yellowish green-colored colonies, and the remaining isolates had dark green-colored colonies. Furthermore, conidiation rates were assessed: 30 isolates were characterized as having low conidiation rates, 15 as having medium conidiation rates, and the remaining 7 isolates 
as having high conidiation rates. Eleven of the isolates appeared to be strongly pathogenic against maize, 15 isolates proved to be weakly pathogenic against maize, and the remaining isolates were regarded to be moderately pathogenic. Interestingly, correlation analysis demonstrated a negative correlation between the growth rate and the pathogenicity of the isolates, while a positive correlation was observed between the conidiation rate and the pathogenicity. No correlation was observed between the colony color and the pathogenicity of the isolates.

Key words: Curvularia lunata; ITS; Genetic divergence; Pathogenicity; Correlation

\section{INTRODUCTION}

Curvularia leaf spot caused by the fungal pathogen Curvularia lunata (Wakker) Boed greatly influences corn cultivation and production worldwide (Dai et al., 1995; Dai et al., 1998; Li et al., 2006; Gao et al., 2014). In China, C. lunata disease has become a major maize disease; in the 1990s, the pathogen caused tremendous yield loss and spread across more than 10 provinces nationwide. The occurrence of this disease in maize, however, has decreased with the cultivation of resistant varieties. Nevertheless, some studies have suggested the emergence of genetic variability in this pathogen with the cultivation of disease-resistant varieties. In support of this, Le et al found that a resistant $C$. lunata variety (Newlron 10) had lost disease resistance in the field, presumably from the emergence of a new, more pathogenic strain of the fungus (Le and Liu, 1997). Genetic diversity studies on a limited number of fungal isolates have furthermore demonstrated significant variability among C. Iunata isolates (Chen et al., 2003; Yan et al., 2005; Xu et al., 2007): 100 genetic polymorphisms were detected in 22 isolates of $C$. lunata using the random amplified polymorphic DNA technique (RPAD) (Yan and Chen, 2002), and 110 reproducible bands and 75 special proteins associated with virulence differentiation were separated from 6 isolates of $C$. lunata by one- and two-dimensional electrophoresis in 2007, indicative of huge biodiversity among the isolates (Xu et al., 2007).

In recent years, the maize cropping system has changed substantially in China with the increased cultivation of disease-resistant varieties and/or cultivation of single, high-yield maize varieties (Chen et al., 2011). These changes may have affected maize pathogen populations including $C$. lunata. However, new information regarding changes in the $C$. lunata population, due to the cultivation of disease-resistant varieties and new cropping systems, is limited.

An internal transcribed spacer (ITS) is a piece of non-functional RNA situated between structural ribosomal RNAs on a common precursor transcript. These RNA species and act as universal DNA barcode markers in fungi. Currently, ITS regions have been widely used in taxonomy and molecular phylogeny because they can be easily amplified from small quantities of DNA, and they furthermore exhibit a high degree of variation between closely related species (Schoch et al., 2012). The main purpose of this study was to assess the genetic relationships between isolates of $C$. Iunata from different maize growing areas in China using ITSs as indicators, and to determine whether new genetic divergence has occurred in this species. A further aim of this study was to gain some insight into the morphological and pathogenic variability among $C$. lunata isolates. 


\section{MATERIAL AND METHODS}

\section{Collection and isolation of fungal isolates}

Maize leaves with spots typical for Curvularia leaf spot infection were collected from at least 3 different districts in each of 13 provinces in China (Heilongjiang, Jilin, Liaoning, Shandong, Henan, Shaanxi, Beijing, Shanxi, Anhui, Hebei, Sichuan, Hunan, and Fujian) from 2011 to 2013. The diseased samples were first placed in paper bags and then in polyethylene bags before being brought to the laboratory, where they were stored at $4^{\circ} \mathrm{C}$ until they were processed for isolation. The fungus was isolated via the single spore isolation method as follows: leaves with necrotic spots were cut into small pieces (5-mm long), surface sterilized with $75 \%$ alcohol for $10 \mathrm{~s}$, then rinsed 3 times in sterilized distilled water. The leaf pieces were placed onto potato dextrose agar (PDA) and incubated in the dark at $28^{\circ} \mathrm{C}$ for 3 days. A small portion of an emerging colony was transferred onto a new petri dish containing PDA and incubated in the dark at $28^{\circ} \mathrm{C}$ for a further 3 days. A small portion of the colony was again transferred to new PDA as mentioned above, yielding a pure culture (Zhang et al., 2008).

\section{Identification of isolates and phylogenetic analysis}

Isolated fungal colonies were first identified as $C$. Iunata based on morphology, specifically in terms of the shapes of the conidia and conidiophores (Manamgoda et al., 2012). To further confirm the identity of the isolates, the ITS regions of the isolates were sequenced. Genomic DNA was extracted as previously described (Liu et al., 2011) and the ITS region was amplified by PCR using the universal primers ITS1 and ITS4 (White et al., 1990). The resulting PCR product was sequenced in both directions after cloning into pMD19-T vector (TaKaRa, Dalian, China). The ITS sequences were confirmed to be from $C$. lunata by the blastn search tool on the NCBI website (https://blast.ncbi.nlm.nih.gov). Three C. lunata strains (CX-3, SW-18, and Fk) obtained prior to 2004 were kindly provided by Professor Jie Chen (Shanghai Jiao Tong University, Shanghai, China) and were subjected to ITS sequence analysis. Representative ITS sequences of known C. lunata isolates were downloaded from the NCBI database. All sequences were aligned and edited manually using the MEGA5 (http://megasoftware.net) tool. Moreover, MEGA5 was used to construct a phylogenetic tree by the neighbor-joining method with minimum evolution criterion and 1000 bootstrap replicates as described by Saitou and Nei (1987).

\section{Radial growth rate, colony color, conidiation rate, and pathogenicity of isolates}

To measuring the radial growth rate of the $C$. lunata isolates, a mycelium plug $(4 \mathrm{~mm})$ from a 7-day-old colony of each isolate was placed onto the middle of a PDA plate and incubated for 7 days at $28^{\circ} \mathrm{C}$ in the dark. Colony diameters were measured at 2 opposite axes and averaged, allowing for the calculation of growth rates per day. Based on these growth rates, the isolates were categorized as slow- $(<0.5 \mathrm{~cm} /$ day $)$, medium- $(0.5-1.0 \mathrm{~cm} /$ day $)$, and fast- $(>1.0 \mathrm{~cm} /$ day $)$ growing. Colony color was visually assessed the top of the petri dishes, and colonies were classified as white, yellowish-green, or dark-green.

For the conidiation assays, isolates were grown on PDA for 7 days at $28^{\circ} \mathrm{C}$ in the dark in a chamber. Ten mycelium plugs were taken from the edge of the media with a 4-mm diameter punch 
and then transferred into a tube containing $2 \mathrm{~mL}$ sterile water. The tube was mixed on a shaker and conidia numbers per $\mathrm{mL}$ were subsequently determined using a hemocytometer. Based on the rate of conidiation, isolates were grouped into 3 categories: low productivity $\left(<5 \times 10^{5} \mathrm{conidia} / \mathrm{mL}\right)$, medium productivity $\left(5-10 \times 10^{5}\right.$ conidia/mL), and high productivity $\left(>10 \times 10^{5}\right.$ conidia/mL).

Pathogenicity testing was conducted using the detached leaf assay. Huangzao-4, a maize inbred line susceptible to $C$. lunata, was used as host. The Huangzao- 4 maize line has been used extensively in commercial hybrid production in China and has been well characterized genetically. All seedlings were grown in pots in a growth chamber at $25^{\circ} \mathrm{C}$ with a $14 \mathrm{~h}$ photoperiod and $60 \%$ relative humidity. The fourth leaves of the susceptible maize seedlings at the seventh leaf stage were cut into $5 \mathrm{~cm}$ fragments and placed into humid petri dishes. The leaf fragments were then inoculated with the $4 \mathrm{~mm}$ diameter mycelium blocks obtained from the 7-day-old fungal cultures, and after being placed in the light at $28^{\circ} \mathrm{C}$ for $72 \mathrm{~h}$, the mycelium blocks were removed and the dimensions (length and width) of the resulting necrotic spots were measured. Four different levels of pathogenicity were determined based on the size of the lesions that formed on the detached leaves: 1, strong pathogenicity (lesion areas $\left.\geq 1.0 \mathrm{~cm}^{2}\right) ; 2$, medium pathogenicity $\left(0.3 \mathrm{~cm}^{2} \leq\right.$ lesion area $\left.<1.0 \mathrm{~cm}^{2}\right) ; 3$, weak pathogenicity $\left(0 \mathrm{~cm}^{2}<\right.$ lesion area $\left.<0.3 \mathrm{~cm}^{2}\right)$; and 4 , no pathogenicity (no lesions). Three independent experiments each with 3 replicates were carried out for this assessment.

\section{Correlation analysis}

To assess potential relationships between the pathogenicity of the isolates and the growth rates, colony colors, and conidiation rates of the isolates, correlation coefficients $(R)$ and twodimensional scatter plots were obtained from data analyses carried out with SPSS $19.0^{\circ}$ statistical software (SPSS, Chicago, USA. Correlations were grouped into different types according to the correlation coefficients $(R)$ : $R<0$, negative correlation; $R=0$, non-linear correlation; $R>0$, positive correlation. $|R|>0.95$ was considered a significant correlation, $|R|>0.8$ a high correlation, $0.5 \leq$ $|R|<0.8$ a medium correlation, $0.3 \leq|R|<0.5$ a low correlation, and $|R|<0.3$ a very low correlation or no correlation (Li et al., 2012).

\section{RESULTS}

\section{Iunata identification and confirmation}

In total, 189 isolates were obtained from maize leaves with necrotic spots typical of $C$. lunata infection (Figure 1a) collected from different maize production sites in China. Based on morphological criteria (Figure 1b, c), 58 isolates were initially considered to potentially be C. lunata; however, a Blastn search with ITS sequences in the GenBank database revealed that only 52 of these 58 isolates were in fact $C$. lunata. Thus, both phylogenetic and biological assay analyses were performed only on the $52 \mathrm{C}$. Iunata isolates identified based on both morphology and ITS sequence analysis.

\section{Phylogenetic analysis}

Phylogenetic analysis of the ITS sequences from 52 isolates, 10 representative C. lunata strains from NCBI GenBank, and 3 C. lunata isolates (CX-3, SW-18, and Fk) revealed 2 major genetic groups (G1 and G2) in C. lunata, supported by high bootstrap values (Figure 2). Group G1 was further 
subdivided into 2 subgroups ( $/$ and II). Subgroup / contained 4 isolates (LD-1, SD-11, AH-7, and AH11) from 3 different provinces and these isolates were clustered into 1 branch with $89 \%$ bootstrap. Subgroup II contained 33 isolates from different provinces as well as 2 isolates (CX-3 and SW18) isolated prior to 2004. Group G2 was subdivided into 3 subgroups (III, IV, and V). The 10 sequences downloaded from GenBank were clustered separately into subgroup III; none of the isolates from this study was nested in subgroup III. Subgroup $I V$ contained 11 isolates collected from 2011 to 2013 and 1 isolate (C. lunata Fk) from 2004. Subgroup $V$ contained 4 isolates (SC-2, SC-6, SC-8, and SC-9) from the same province. Our data showed 8 isolates (LD-1, SD-11, AH-7, AH-11, SC-2, SC-6, SC-8, and SC-9) from this isolation to be divided into 2 separate branches compared with strains isolated before 2004 , suggesting that the pathogen C. lunata did undergo new genetic divergence.
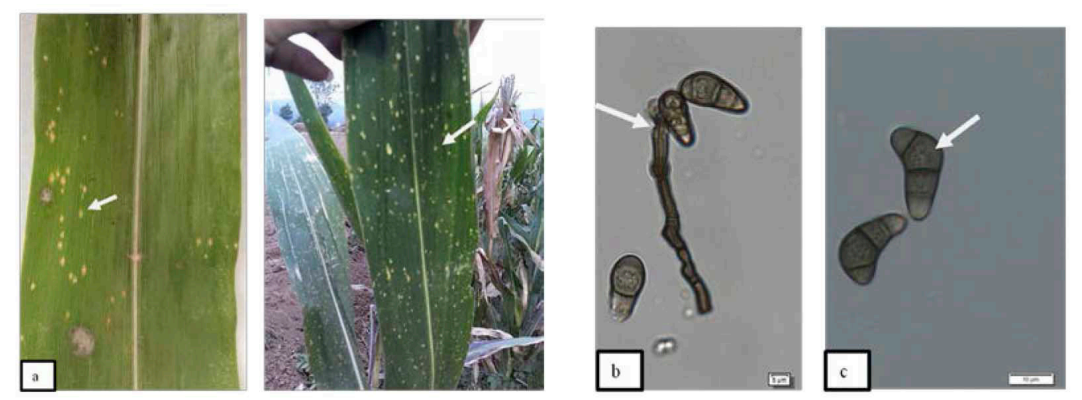

Figure 1. Maize leaves with necrotic spots typical of Curvularia lunata infection (a) and characteristic conidiophores (b) and conidia (c) of C. lunata.

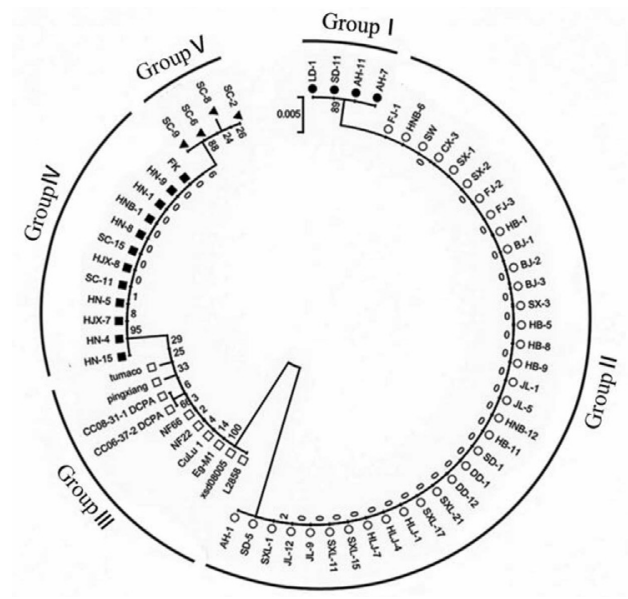

Figure 2. Unrooted phylogenetic tree representing the relationships among Curvularia lunata isolates. The ITS sequences of the isolates in this study were aligned along with representative $C$. Iunata ITS sequences from GenBank using ClustalW ${ }^{\odot}$ program. The phylogenetic tree was constructed using the neighbor-joining method in MEGA 5.0, and the neighbor-joining tree was drawn with the TreeView program. Bootstrap vales are indicated at the branch. Five groups (I to IV) are indicated by black circle. The ITS sequences of the 52 study isolates as well as those of three other isolates (CX-3, SW-18, and Fk) were submitted to GenBank (accession No. 5042709). The GenBank accession No. of known isolates downloaded from the NCBI database were as follows: L2858 (508078110); xsd08005 (225675530); pingxiang (387158753); tumaco (310659896); Eg-M1 (616996382); CC06-37-2_DCPA (478432581); CC08-31-1_DCPA (478432580); CuLu 1(567556081); NF22 (646281764); NF66 (646281765). 


\section{Biological characteristics}

The biological characteristics (radial growth rate, colony color, conidiation rate, and pathogenicity) of the 52 isolates are shown in Table 1. Significant differences were observed between the growth rates of the $52 \mathrm{C}$. lunata isolates collected from different districts. Isolate HLJ1 showed the highest growth rate $(1.41 \pm 0.01 \mathrm{~cm} /$ day $)$, while isolate HB-11 showed lowest growth rate $(0.35 \pm 0.02 \mathrm{~cm} /$ day $)$. Of the total of 52 isolates, 7 isolates showed radial growth rates above $1.0 \mathrm{~cm} /$ day, while the growth rates of 32 isolates were found to be $<0.5 \mathrm{~cm} /$ day. The remaining isolates showed growth rates of $0.5-1.0 \mathrm{~cm} /$ day. The individual radial growth rates of all isolates are shown in (Table 1, column 3). In addition, significant differences were observed in the colony colors of the 52 isolates. The $C$. Iunata isolates were cultured on PDA plates for 7 days in the dark at $28^{\circ} \mathrm{C}$ and colony color was then assessed visually using a standard color chart. Three colony colors were observed: white, yellowish-green, and dark-green with 3, 13, and 36 isolates being grouped into each of these color types, respectively (Table 1, column 4). The differences between the conidial production rates of the isolates were found to be highly significant and the rates ranged from 0.06 $\pm 0.01 \times 10^{5}$ conidia $/ \mathrm{mL}$ to $13.61 \pm 1.55 \times 10^{5} \mathrm{conidia} / \mathrm{mL}$. Analysis of the conidial production data revealed that 7 isolates had conidial production rates above $10 \times 10^{5}$ conidia/mL (considered high conidial productivity), while 30 isolates exhibited low conidial production rates of below $5 \times 10^{5}$ conidia/mL. The rest of the isolates (conidial production rates $5-10 \times 10^{5}$ conidia/mL) were grouped as having medium conidial productivity (Table 1, column 5). Furthermore, significant variations in pathogenicity with regard to the lesions formed on detached leaves were observed among the 52 isolates. Eleven isolates were found to induce strong reactions on detached leaves, resulting in lesion areas $\geq 1.0 \mathrm{~cm}$. These were classed as strongly pathogenic. Fifteen isolates only induced a weak reaction in detached leaves with resulting lesion areas between 0 and $0.3 \mathrm{~cm}^{2}$; these were classed as weakly pathogenic. The remaining isolates appeared to have medium pathogenicity, with lesion areas between 0.3 and $1 \mathrm{~cm}^{2}$ (Table 1, column 6).

\section{Correlation analysis: biological characteristics and pathogenicity}

Two-dimensional scatter plots of the relationships between biological characteristics and pathogenicity were established using SPSS $19.0^{\circ}$ software. The results demonstrated a negative correlation between the growth rates and pathogenicities of the isolates with correlation coefficient $(R)=-0.433$ and significance probability $(P)=0.001$ (Figure 3a). A positive correlation was observed between the conidiation rates and pathogenicities of the isolates $(R=0.476, P=0.000$, Figure $3 b)$, while no correlation was observed between the colony colors and the pathogenicities of the isolates $(R=0.164, P=0.245$, Figure $3 c)$.

\section{DISCUSSION}

Pathogens that cause Curvularia leaf spot in maize include C. lunata, C. pallescens, C. maculans, C. clavata, C. tetramera, C. inaequalis, and C. senegalensis (Shi et al., 2000). Among them, $C$. lunata is considered the major pathogen. It is very difficult to identify these closely related pathogenic species based on culture characteristics such as spore shape and colony appearance because many of these species share similar characteristics (Manamgoda et al., 2012). In this study, 58 isolates were initially thought to be $C$. lunata based on the conidia and conidiophore 


\begin{tabular}{|c|c|c|c|c|c|}
\hline Isolate code & Origin of isolate & Growth rate (cm/day) & Colony color & Conidiation $10^{5} / \mathrm{mL}$ & Lesion area $\left(\mathrm{cm}^{2}\right)$ \\
\hline HNB-1 & Henan, Zhenzhou & $1.19 \pm 0.01$ & White & $3.11 \pm 0.33$ & $0.68 \pm 0.03$ \\
\hline HNB-6 & Henan, Xinyang & $1.07 \pm 0.02$ & Dark green & $2.50 \pm 0.38$ & $0.57 \pm 0.03$ \\
\hline HNB-12 & Henan, Xinxiang & $1.13 \pm 0.02$ & Dark green & $4.28 \pm 0.65$ & $0.41 \pm 0.01$ \\
\hline $\mathrm{AH}-1$ & Anhui, Huaibei & $1.05 \pm 0.01$ & Yellow green & $2.72 \pm 0.28$ & $0.38 \pm 0.04$ \\
\hline $\mathrm{AH}-7$ & Anhui, Suzhou & $1.12 \pm 0.03$ & Dark green & $2.50 \pm 0.62$ & $0.35 \pm 0.02$ \\
\hline $\mathrm{AH}-11$ & Anhui, Buyang & $1.18 \pm 0.01$ & Dark green & $5.61 \pm 1.39$ & $0.38 \pm 0.01$ \\
\hline LD-1 & Liaoli, Shenyang & $1.07 \pm 0.03$ & Dark green & $8.61 \pm 0.89$ & $0.76 \pm 0.04$ \\
\hline DD-1 & Liaoli, Dandong & $1.19 \pm 0.02$ & Dark green & $2.11 \pm 0.41$ & $0.30 \pm 0.01$ \\
\hline DD-12 & Liaoli, Faku & $0.93 \pm 0.01$ & Dark green & $2.33 \pm 0.65$ & $0.42 \pm 0.03$ \\
\hline$J L-1$ & Jilin, Changchun & $1.14 \pm 0.02$ & Dark green & $6.28 \pm 0.85$ & $0.51 \pm 0.02$ \\
\hline JL-5 & Jilin, Siping & $1.11 \pm 0.01$ & Dark green & $2.11 \pm 0.25$ & $0.36 \pm 0.02$ \\
\hline JL-9 & Jilin, Baicheng & $1.08 \pm 0.03$ & Dark green & $10.06 \pm 0.79$ & $1.09 \pm 0.04$ \\
\hline JL-12 & Jilin, Songyuan & $1.06 \pm 0.02$ & Dark green & $2.94 \pm 0.41$ & $0.79 \pm 0.03$ \\
\hline SXI-1 & Shanxi, Xianyang & $1.05 \pm 0.03$ & Yellow green & $6.00 \pm 0.53$ & $0.80 \pm 0.04$ \\
\hline SXI-11 & Shanxi, Baoji & $0.96 \pm 0.02$ & Dark green & $8.89 \pm 0.58$ & $1.07 \pm 0.03$ \\
\hline SXI-15 & Shanxi, Ankang & $0.91 \pm 0.02$ & Dark green & $5.39 \pm 0.39$ & $0.25 \pm 0.01$ \\
\hline SXI-17 & Shanxi, Yangli & $1.12 \pm 0.01$ & Yellow green & $1.94 \pm 0.24$ & $0.23 \pm 0.02$ \\
\hline SXI-21 & Shanxi, Weinan & $1.04 \pm 0.01$ & Dark green & $3.11 \pm 0.53$ & $0.96 \pm 0.03$ \\
\hline SD-1 & Shandong, Dai'an & $1.08 \pm 0.03$ & Yellow green & $4.94 \pm 0.65$ & $0.43 \pm 0.02$ \\
\hline SD-5 & Shandong, Dongying & $1.05 \pm 0.02$ & Yellow green & $4.94 \pm 0.52$ & $0.45 \pm 0.02$ \\
\hline SD-7 & Shandong, Liaocheng & $0.63 \pm 0.02$ & Yellow green & $3.74 \pm 0.49$ & $1.04 \pm 0.03$ \\
\hline SD-11 & Shandong, Heze & $0.84 \pm 0.02$ & Yellow green & $2.89 \pm 0.49$ & $0.45 \pm 0.02$ \\
\hline HB-1 & Hebei, Shijiazan & $1.21 \pm 0.02$ & Dark green & $9.33 \pm 0.96$ & $1.10 \pm 0.02$ \\
\hline HB-5 & Hebei, Baoding & $1.27 \pm 0.02$ & Dark green & $3.61 \pm 0.48$ & $0.16 \pm 0.02$ \\
\hline HB-8 & Hebei, Hanshui & $1.33 \pm 0.04$ & Dark green & $10.22 \pm 2.03$ & $0.21 \pm 0.01$ \\
\hline HB-9 & Hebei, Handan & $0.37 \pm 0.01$ & Dark green & $13.61 \pm 1.55$ & $1.61 \pm 0.03$ \\
\hline HB-11 & Hebei, Qifangdao & $0.35 \pm 0.02$ & Dark green & $13.06 \pm 1.68$ & $1.68 \pm 0.03$ \\
\hline HLJ-1 & Heilongjiang, Daqing & $1.41 \pm 0.01$ & Dark green & $10.67 \pm 0.97$ & $0.10 \pm 0.01$ \\
\hline HLJ-4 & Heilongjiang, Harbin & $1.00 \pm 0.02$ & Yellow green & $7.25 \pm 2.32$ & $0.35 \pm 0.02$ \\
\hline \multirow[t]{2}{*}{ HJX-7HJX-8 } & Heilongjiang, Jiamusi & $0.65 \pm 0.03$ & Dark green & $2.11 \pm 0.25$ & $0.83 \pm 0.01$ \\
\hline & Heilongjiang, Mudanjiang & $1.00 \pm 0.03$ & Dark green & $6.28 \pm 1.46$ & $0.37 \pm 0.02$ \\
\hline $\mathrm{HN}-1$ & Hunan, Shaoyang & $0.91 \pm 0.02$ & Dark green & $4.83 \pm 1.25$ & $0.18 \pm 0.02$ \\
\hline $\mathrm{HN}-4$ & Hunan, Changde & $1.10 \pm 0.04$ & Dark green & $2.97 \pm 1.22$ & $0.39 \pm 0.04$ \\
\hline $\mathrm{HN}-5$ & Hunan, Loudi & $0.99 \pm 0.02$ & Dark green & $10.28 \pm 1.49$ & $1.24 \pm 0.02$ \\
\hline $\mathrm{HN}-8$ & Hunan, Changsha & $0.77 \pm 0.03$ & Yellow green & $6.39 \pm 0.79$ & $0.34 \pm 0.02$ \\
\hline $\mathrm{HN}-9$ & Hunan, Zhuzhou & $0.39 \pm 0.01$ & Yellow green & $6.17 \pm 1.36$ & $1.20 \pm 0.02$ \\
\hline $\mathrm{HN}-15$ & Hunan, Xiangtan & $0.47 \pm 0.01$ & Yellow green & $5.22 \pm 0.50$ & $0.34 \pm 0.03$ \\
\hline FJ-1 & Fujiang, Fuzhou & $0.64 \pm 0.02$ & Dark green & $0.30 \pm 0.05$ & $0.15 \pm 0.02$ \\
\hline FJ-2 & Fujiang, Zhangzhou & $0.86 \pm 0.03$ & Dark green & $0.13 \pm 0.03$ & $0.12 \pm 0.01$ \\
\hline FJ-3 & Fujiang, Sanming & $1.22 \pm 0.01$ & Dark green & $2.78 \pm 0.53$ & $0.23 \pm 0.03$ \\
\hline BJ-1 & Beijing & $0.91 \pm 0.02$ & Dark green & $6.28 \pm 0.80$ & $0.40 \pm 0.01$ \\
\hline BJ-2 & Beijing & $0.82 \pm 0.02$ & Dark green & $0.45 \pm 0.08$ & $0.28 \pm 0.04$ \\
\hline BJ-3 & Beijing & $0.85 \pm 0.03$ & Dark green & $5.33 \pm 0.69$ & $0.18 \pm 0.01$ \\
\hline SC-2 & Sichuan, Yaan & $1.13 \pm 0.01$ & Yellow green & $4.33 \pm 0.86$ & $0.25 \pm 0.03$ \\
\hline SC-6 & Sichuan, Chendu & $1.01 \pm 0.02$ & Dark green & $5.83 \pm 1.54$ & $0.22 \pm 0.02$ \\
\hline SC-8 & Sichuan, Mianyang & $0.90 \pm 0.02$ & Dark green & $2.72 \pm 0.29$ & $0.64 \pm 0.03$ \\
\hline SC-9 & Sichuan, Huiyang & $0.88 \pm 0.02$ & Dark green & $11.17 \pm 0.84$ & $1.35 \pm 0.02$ \\
\hline SC-11 & Sichuan, Ziyang & $1.24 \pm 0.03$ & White & $0.06 \pm 0.01$ & $0.10 \pm 0.01$ \\
\hline SC-15 & Sichuan, Meishan & $0.92 \pm 0.04$ & White & $0.09 \pm 0.02$ & $0.11 \pm 0.01$ \\
\hline SX-1 & Shanxi, Taiyuan & $1.09 \pm 0.02$ & Dark green & $2.22 \pm 0.42$ & $1.43 \pm 0.01$ \\
\hline$S X-2$ & Shanxi, Changzhi & $0.95 \pm 0.04$ & Dark green & $2.00 \pm 0.14$ & $1.20 \pm 0.04$ \\
\hline SX-3 & Shanxi, Jizhong & $0.40 \pm 0.05$ & Yellow green & $0.12 \pm 0.03$ & $0.93 \pm 0.04$ \\
\hline
\end{tabular}

Data are reported as means \pm SD from three independent experiments with three replicates and were analyzed using SPSS 19.0 statistical software (SPSS, Chicago, IL).

shapes; however, only 52 of these isolates were finally confirmed to be $C$. lunata based on analysis of ITS sequences. These findings confirm that molecular-based identification is indeed the gold standard for the accurate identification of $C$. lunata. Previous studies have demonstrated the ITS region to be very useful in the identification and assessment of genetic relationships among fungi, 
because the ITS region of fungi exhibits abundant diversity even within species (Schoch et al., 2012). In this study, the ITS sequencing data allowed the C. lunata isolates to be clustered into 2 distinct clusters with several subgroups within each genetic group, indicating a high level of genetic diversity within $C$. lunata. To assess whether new genetic divergence has emerged in $C$. lunata since 2004 , ITS sequence data of $C$. lunata strains was downloaded from GenBank (NCBI) for comparison, and the ITS regions of additional strains (CX-3, SW-18, and Fk) obtained prior to 2004 were sequenced. Including these additional data, the phylogenetic tree was still found to be divided into 2 large clades. Eight isolates (LD-1, SD-11, AH-7, AH-11, SC-2, SC-6, SC-8, and SC-9), however, formed a new subclade. These findings suggest that new genetic divergence had occurred in C. lunata.
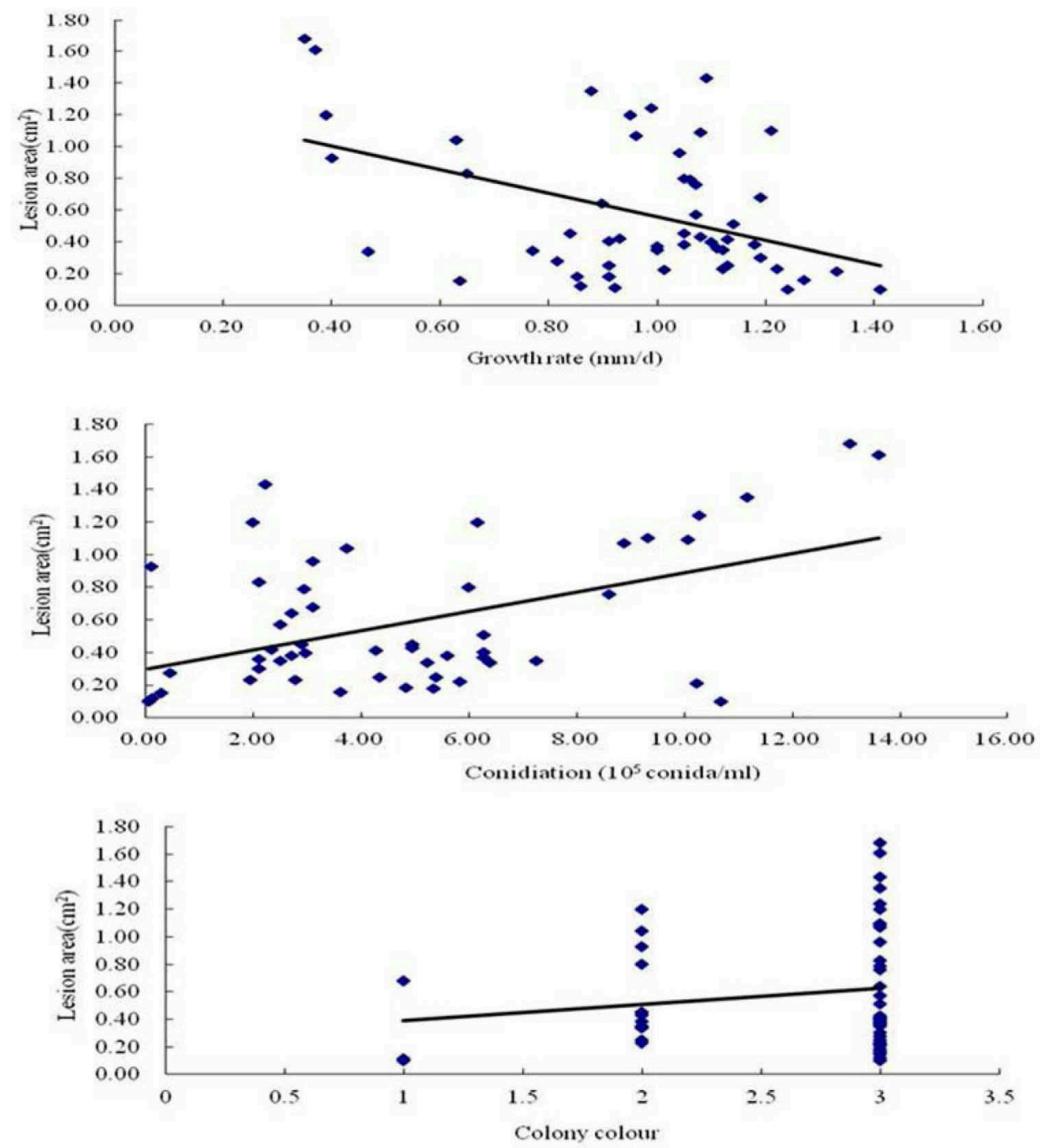

Figure 3. Correlations between the pathogenicities of the 52 isolates and their radial growth rates (a), conidiation rates (b), and colony colors (c) represented as scatterplot charts. 
Potential correlations between the pathogenicities and selected biological characteristics (conidiation rate, growth rate, and colony color) of the 52 isolates were assessed using SPSS $19.0^{\odot}$ software. A negative correlation between the fungal growth rate and the pathogenicity of the $C$. lunata isolates was observed, indicating that slow-growing isolates did not exhibit reduced pathogenicity - in fact, these isolates exhibited strong pathogenicity. The fast-growing isolates SXI-17, HB-5, HB-8, HLJ-1, FJ-3, SC-2, SC-6, and SC-11, for instance, were found to be only weakly pathogenic, while the slow-growing isolates HB-9, HB-11, and HN-9 were found to be highly pathogenic. The pathogenicity of a slow-growing $C$. lunata mutant obtained by Agrobacteriummediated transformation to detached maize leaf was previously shown not to differ markedly from that of the wild-type strain (Liu et al., 2011). This phenomenon suggests that there is no clear link between the genes that control growth and those that determine pathogenicity in C. lunata. A positive correlation was observed between the conidiation rates and the pathogenicities in the 52 isolates, indicative of conidiation rate affecting the pathogenicity of $C$. lunata. Conidiation-related genes may therefore be linked to some extent with pathogenicity-related genes. This hypothesis is further supported by the results of various other studies on plant fungi (Yi et al., 2008; Kim et al., 2011). In the present study, no relationship was observed between the colony color and the pathogenicity of the isolates. Among the dark-green isolates, SXI-15, HB-8, HLJ-1, FJ-2, FJ-3, BJ-2, BJ-3, and SC-6 exhibited low pathogenicity, while isolates JL-9, SXI-11, HB-1, HB-9, HB$11, \mathrm{HN}-5, \mathrm{SC}-9$, SX-1, and SX-2 exhibited strong pathogenicity. Similarly, the white isolate HNB1 was moderately pathogenic, while the white isolates SC-11 and SC-15 exhibited only weak pathogenicity. These findings contradict some of our previous findings (Liu et al., 2011). Melanin in C. lunata is known to consolidate the structures of the pathogen, such as appressoria and infection pegs, that are required for effective mechanical penetration. According to current understanding, C. lunata might produce other virulence factors such as cell wall-degrading enzymes, toxins, and laccase (Feng et al., 2002; Zhou et al., 2010). Evidence indicates that the pigment produced by a pathogen is not the main factor determining pathogenicity, but rather that the pigment is a result of diversified environmental behavior. It is possible that the results of the pathogenicity versus color correlation analysis in this study were affected by different division standards; however, these data will nevertheless provide some useful information for further study on C. lunata.

\section{Conflicts of interest}

The authors declare no conflict of interest.

\section{ACKNOWLEDGMENTS}

Research supported by the Nature Science Foundation of Heilongjiang Province (Grant \#C201448), the National Key Technology R\&D Program (\#2104BAD07B05) and Key Science Foundation of Heilongjiang Agricultural Reclamation (\#HNK125B-03-02).

\section{REFERENCES}

Chen J, Yan H, Gao Z, Xue C, et al. (2003). Identification techniques for physiological differentiation of Curvularia lunata in maize. Acta Phytopathol. Sin. 33: 121-125.

Chen XP, Cui ZL, Vitousek PM and Cassman KG, et al. (2011). Integrated soil-crop system management for food security. Proc. Natl. Acad. Sci. U. S. A. 108: 6399-6404. 
Dai FC, Gao WD, Wu RJ and Jin XH (1995). A noticeable corn disease: Curvularia leaf spot. Acta Phytopathol. Sin. $25: 330$.

Dai FC, Wang XM, Zhu ZD, Gao WD, et al. (1998). Curvularia leaf spot of maize: pathogens and varietal resistance. Acta Phytopathol. Sin. 2: 123-129.

Feng J, Gao ZG, Xue CS, Zhuang JH, et al. (2002). The pathogenesis of the cell-degrading enzymes produced by Curvularia lunata. Rain Fed Crops 22: 164-166.

Gao JX, Liu T and Chen J (2014). Insertional mutagenesis and cloning of the gene required for the biosynthesis of the non-hostspecific toxin in Cochliobolus lunatus that causes maize leaf spot. Phytopathology 104: 332-339.

Kim H, Smith JE, Ridenour JB, Woloshuk CP, et al. (2011). HXK1 regulates carbon catabolism, conidiation, fumonisin B1 production and pathogenesis in Fusarium verticillioides. Microbiology 157: 2658-2669.

Le GZ and Liu ZH (1997). A new disease-Curvularia leaf spot of corn by Curvularia lunata. J. Shenyang Agric. Univ. 28: 75-76.

Li JT, Fu JF, Yan XR, Li HC, et al. (2006). Analysis of temporal dynamics of Curvularia leaf spot of maize (Curvularia lunata) epidemic and yield loss. J. Shenyang Agric. Univ. 6: 835-839.

Li Y, Yu JJ, Liu YF, Xiao L, et al. (2012). Determination of conidiation and pathogenicity of Ustilaginoidea virens. China Agric. Sci. 45: 4166-4177.

Liu T, Xu SF, Liu LL, Zhou FH, et al. (2011). Functional analysis of multi-copy Brn1 gene from the phytopathogenic fungus Curvularia lunata. Eur. J. Plant Pathol. 131: 211-219.

Manamgoda DS, Cai L. McKenzie EHC, Crous PW, et al. (2012). A phylogenetic and taxonomic re-evaluation of the BipolarisCochliobolus-Curvularia complex. Fungal Divers. 56: 131-144.

Schoch CL, Seifert KA, Huhndorf S, Robert V, et al. (2012). Nuclear ribosomal internal transcribed spacer (ITS) region as a universal DNA barcode marker for fungi. Proc. Natl. Acad. Sci. U. S. A. 109: 6241-6246.

Shi J, Liu YY and Wei LN (2000). On pathogens of maize Curvularia leaf spot. J. Shenyang Agric. Univ. 31: $479-481$.

Saitou N and Nei M (1987). The neighbor-joining method: a new method for reconstructing phylogenetic trees. Mol. Biol. Evol. 4: 406-425.

White TJ, Bruns T, Lee S and Taylor J (1990). Amplification and direct sequencing of fungi ribosomal RNA genes for phylogenetics. In: PCR protocols: A guide to methods and applications (Innis MA, Gelfand DH, Sninsky JJ and White TJ, eds.). Academic Press, New York, 315-322.

Xu SF, Chen J, Liu LX, Wang X, et al. (2007). Proteomics associated with virulence differentiation of Curvularia lunata in maize in China. J. Integr. Plant Biol. 49: 487-496.

Yan $\mathrm{HH}$ and Chen $\mathrm{J}$ (2002). Physiological differentiation and molecular biology of Curvularia lunata in maize leaf spot. Acta Phytopathol. Sin. 32: 288.

Yan HH, Chen J, Gao Z, Xia S, et al. (2005). The heredity and variation of the interaction between Curvularia lunata and plant. J. Maize Sci. 13: 119-120.

Yi M, Park JH, Ahn JH and Lee YH (2008). MoSNF1 regulates sporulation and pathogenicity in the rice blast fungus Magnaporthe oryzae. Fungal Genet. Biol. 45: 1172-1182.

Zhang $\mathrm{H}$, Zhang Z, Xu JS, Xu J, et al. (2008). A rapid and simple method for obtaining single-spore isolates of Fusarium species-agar dilution lineation separation. Plant Protect. 6: 134-136.

Zhou SY, Yang HY, Zhao M and Mei LY (2010). The pathogen identification of maize Curvularia leaf spot and analysis on the enzyme activity of the cell wall-degrading enzyme and lactase. J. Maize Sci. 18: 126-130. 